\section{C-Terminal HIV-1 Transframe p6* Tetrapeptide Blocks Enhanced Gag Cleavage Incurred by Leucine Zipper Replacement of a Deleted p6* Domain}

\author{
Fu-Hsien Yu, a,b Kuo-Jung Huang, ${ }^{b}$ Chin-Tien Wang a,b \\ Department of Medical Research, Taipei Veterans General Hospital, a and Institute of Clinical Medicine, National \\ Yang-Ming University School of Medicine, , Taipei, Taiwan
}

ABSTRACT HIV-1 protease (PR) functions as a homodimer mediating virus maturation following virus budding. Gag-Pol dimerization is believed to trigger embedded PR activation by promoting PR dimer formation. Early PR activation can lead to markedly reduced virus yields due to premature Gag cleavage. The p6* peptide, located between Gag and PR, is believed to ensure virus production by preventing early PR maturation. Studies aimed at finding supporting evidence for this proposal are limited due to a reading frame overlap between $p 6^{*}$ and the p6gag budding domain. To determine if $p 6^{*}$ affects virus production via the modulation of PR activation, we engineered multiple constructs derived from Dp6*PR (an assembly- and processing-competent construct with Pol fused at the inactivated PR C terminus). The data indicated that a $\mathrm{p} 6^{*}$ deletion adjacent to active PR significantly impaired virus processing. We also observed that the insertion of a leucine zipper (LZ) dimerization motif in the deleted region eliminated virus production in a PR activitydependent manner, suggesting that the $L Z$ insertion triggered premature PR activation by facilitating PR dimer formation. As few as four C-terminal $p 6^{*}$ residues remaining at the $\mathrm{p} 6^{*} / \mathrm{PR}$ junction were sufficient to restore virus yields, with a Gag processing profile similar to that of the wild type. Our study provides supporting evidence in a virus assembly context that the C-terminal $p 6^{*}$ tetrapeptide plays a role in preventing premature PR maturation.

IMPORTANCE Supporting evidence for the assumption that $\mathrm{p} 6^{*}$ retards PR maturation in the context of virus assembly is lacking. We found that replacing $p 6^{*}$ with a leucine zipper peptide abolished virus assembly due to the significant enhancement of Gag cleavage. However, as few as four C-terminal $\mathrm{p}^{*}$ residues remaining in the deleted region were sufficient for significant PR release, as well as for counteracting leucine zipperincurred premature Gag cleavage. Our data provide evidence that (i) $p 6^{*}$ ensures virus assembly by preventing early PR activation and (ii) four C-terminal $6^{*}$ residues are critical for modulating PR activation. Current PR inhibitor development efforts are aimed largely at mature PR, but there is a tendency for HIV-1 variants that are resistant to multiple protease inhibitors to emerge. Our data support the idea of modulating PR activation by targeting PR precursors as an alternative approach to controlling HIV-1/AIDS.

KEYWORDS Gag, human immunodeficiency virus, protease, virus assembly

D uring or soon following HIV-1 virion release from cells, the viral capsid precursor Pr55gag is cleaved by viral protease (PR) into four domains: matrix (MA) (p17), capsid (CA) (p24), nucleocapsid (NC) (p7), and C-terminal p6 (1). SP1 (p2gag) and SP2 (p1gag) spacer peptides separate NC from CA and p6gag, respectively. PR is encoded by $\mathrm{pol}$, which is initially translated as a Gag-Pol fusion polyprotein. Gag-Pol dimerization is believed to trigger PR activation. Once activated, PR functions as a homodimer,
Received 18 January 2017 Accepted 15 February 2017

Accepted manuscript posted online March 2017

Citation YU F-H, Huang K-J, Wang C-T. 2017 C-terminal HIV-1 transframe $6^{*}$ tetrapeptide blocks enhanced Gag cleavage incurred by leucine zipper replacement of a deleted $^{*}$ domain. J Virol 91:e00103-17. https://doi.org/ 10.1128/JVI.00103-17.

Editor Frank Kirchhoff, Ulm University Medical Center

Copyright $\odot 2017$ American Society for Microbiology. All Rights Reserved. Address correspondence to Chin-Tien Wang, chintien@ym.edu.tw. 
cleaves from Gag-Pol, and mediates Gag and Gag-Pol polyprotein processing (2). Three pol-encoded viral enzymes emerge from Gag-Pol cleavage: PR, reverse transcriptase (RT), and integrase (IN). PR-mediated Gag and Gag-Pol processing (referred to as virus maturation) is essential for viral infectivity acquisition (1, 3-6).

Both Gag and Gag-Pol are translated from the same mRNA template. During Gag translation, a -1 ribosomal shift to a Pol reading frame occurs at a frequency of $5 \%$, resulting in a Gag-Pol/Gag expression ratio of approximately 1/20 (7). Within Gag-Pol, p1-p6gag is truncated and replaced with a p6pol or $6^{*}$ transframe region (TFR). Maintaining a low level of Gag-Pol expression is critical for virus assembly, since any overexpression of Gag-Pol leads to markedly reduced virus yields due to enhanced Pr55gag cleavage (8-14). Equally important for virus assembly is the timing of PR activation, since premature activation results in a significant reduction in virus yield due to premature Gag cleavage. This hypothesis is supported by observations that either Gag-Pol dimerization as facilitated by a RT dimerization enhancer or PR dimerization facilitated by the placement of a leucine zipper (LZ) motif at the PR C terminus leads to significant decreases in virus production (15-17). Accordingly, the prevention of premature PR-mediated virus maturation appears to be an important aspect of virus assembly.

The $\mathrm{p}^{*}$ transframe region has been proposed to play a key role in PR activity regulation. The removal of $p 6^{*}$ from the PR precursor $\left(p 6^{*}-P R\right)$ is essential for full PR function (18-21). Further, p6*-derived peptides can inhibit PR activity $(22,23)$, and the presence of $\mathrm{p} 6^{*}$ between duplicated PR domains reduces PR activity (24). Evidence in support of the $p 6^{*}$ transframe region assumption is the significant enhancement of Gag-Pol autoprocessing that follows the complete removal of p6* (19). These data suggest a negative role of $\mathrm{p} 6^{*}$ in PR activation regulation.

Besides lacking a specific structure, p6* exhibits a drug-resistant polymorphism and multiple minor deletions or insertions in natural HIV-1 isolates (25-30). Fully functional HIV-1 PR requires a mature and stable dimeric structure. Molecular models suggest that $\mathrm{p} 6^{*}$ is capable of preventing premature PR activation by destabilizing the folded PR dimer structure (31-34). Since Gag-Pol dimerization triggers PR activation, it is possible

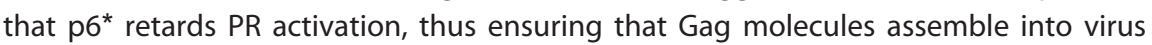
particles prior to premature PR cleavage. However, to date no evidence has been offered in support of this assumption in the context of virus assembly.

We found that $\mathrm{p}^{*}$ replacement with a leucine zipper (LZ) dimerization motif abolished virus assembly as a result of the significant enhancement of PR-mediated Gag cleavage. This LZ-incurred elimination of virus assembly was substantially reduced when as few as four C-terminal $p 6^{*}$ amino acid residues were present between the LZ and PR. Our data provide evidence that $\mathrm{p}^{*}$ ensures virus assembly by preventing premature PR activation triggered by a potent upstream dimerization motif.

\section{RESULTS}

Replacement of p6* with an LZ motif blocks virus production. Since genetic analyses of $\mathrm{p}^{*}$ function with viral backgrounds are restricted due to overlapping $\mathrm{p} 6^{*}$ and p1-p6gag reading frames, we adopted a Gag/Gag-Pol expression plasmid created by placing the Pol at the $C$ terminus of an inactivated PR (24). This construct (designated Dp6*PR) expressed Gag-Pol containing duplicated p6*-PR, with the first PR copy being intentionally mutated to remove enzymatic activity. Given that PR dimer interaction enhancement facilitates PR maturation, the $\mathrm{p} 6^{*}$ substitution with a dimerization motif may trigger premature PR activation by facilitating PR dimer interaction. Alternatively, $\mathrm{p}^{*}$ replacement with a foreign dimerization motif may interfere with PR activation, thus impairing virus maturation. To address these issues, $\mathrm{p} 6^{*}$ adjacent to the active PR in Dp6*PR was replaced with a leucine zipper (LZ) dimerization motif, with the resulting construct designated DWzPR (Fig. 1A).

Transient-expression results indicate that $\mathrm{Dp} 6{ }^{*} \mathrm{PR}$ can assemble and process virus particles in a profile similar to that of the wild type (wt) (Fig. 1B, lane 3). In contrast, virus-associated Gag products were barely detectable in DWzPR transfectant superna- 
A
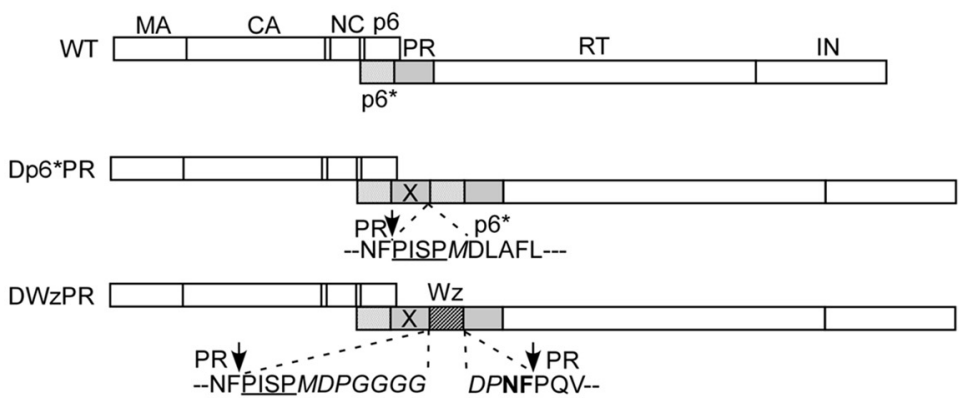

B

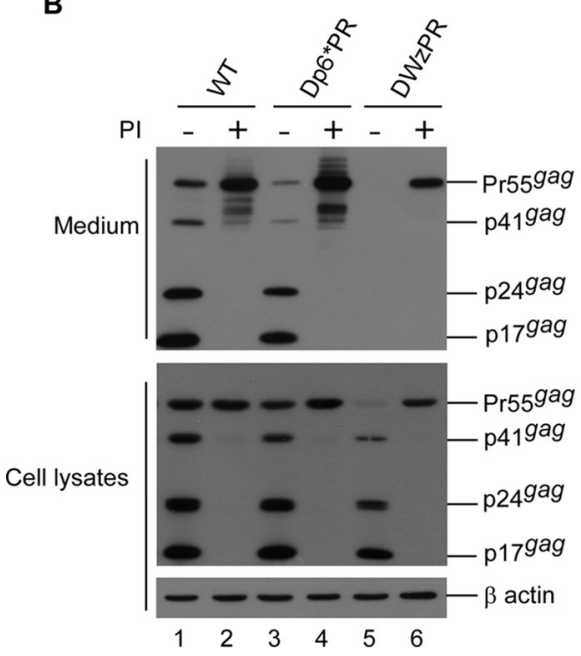

C
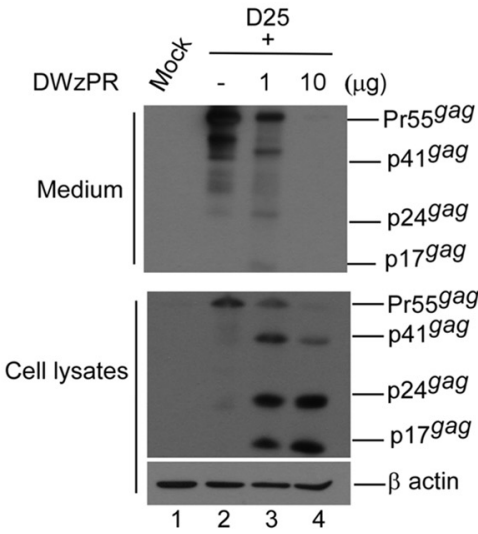

FIG 1 Replacement of $p 6^{*}$ with a leucine zipper motif eliminates virus-like particle production. (A) Schematic representations of HIV-1 Gag and Gag-Pol expression constructs. Indicated are the HIV-1 Gag protein domains MA (matrix), CA (capsid), NC (nucleocapsid), p6, pol-encoded p6* , PR, RT, and IN. The "X" denotes a PR-inactivated mutation. Arrows indicate PR cleavage sites. Underlined "PISP" indicates remaining N-terminal RT residues. The striped (Wz) box denotes wild-type (wt) leucine zipper (LZ). Remaining C-terminal p6* residues are in boldface. Note the deletion of four $\mathrm{N}$-terminal residues in the second p6* copy. Altered or additional residues are in italic. (B) The DWzPR assembly defect is protease activity dependent. 293T cells were transfected with the designated constructs. At $4 \mathrm{~h}$ posttransfection, equal amounts of cells were plated on two dishes and either left untreated or treated with saquinavir (an HIV-1 protease inhibitor) at a concentration of $5 \mu \mathrm{M}$. Supernatants and cells were collected at $48 \mathrm{~h}$ posttransfection, prepared, and subjected to Western immunoblotting. (C) Transdominant inhibition of HIV-1 Gag particle production. The indicated amounts of DWzPR plasmids were coexpressed with $10 \mu \mathrm{g}$ of an HIV-1 protease-defective (D25) Gag/Gag-Pol expression vector. At $48 \mathrm{~h}$ posttransfection, cells and supernatants were collected and analyzed by Western immunoblotting. HIV-1 Gag proteins were probed with anti-p17MA and anti-p24CA monoclonal antibodies. Indicated are the HIV-1 Pr55gag, p41gag, p24gag, and p17gag positions.

tants in repeat independent experiments. Presumably this was due to enhanced Gag cleavage by PR, since virus-associated Pr55gag became readily detected when PR activity was reduced by a PR inhibitor (Fig. 1B, lane 5 versus lane 6). DWzPR was also capable of efficiently cleaving coexpressed Pr55gag from a PR-inactivated (D25) mutant, significantly reducing D25 virus yields in a dose-dependent manner (Fig. 1C). Combined, these data suggest that the LZ replacement of p6* may facilitate PR maturation, resulting in the considerable enhancement of Gag cleavage.

Carboxyl-terminal p6* residues reduce the LZ-induced enhancement effect on Gag cleavage. If enhanced Gag cleavage resulted from PR dimer interactions facilitated by the inserted leucine zipper (LZ), the replacement of $p 6^{*}$ with a motif lacking dimerization ability would likely fail to enhance Gag cleavage efficiency. To test this hypothesis, we created DKzPR by replacing $p 6^{*}$ with a dimerization-defective LZ mutant. Within both DWzPR and DKzPR, only two C-terminal p6* residues remained between the LZ and the active PR. Since C-terminal p6* residues are involved in the PR maturation process, constructs containing the four and six remaining C-terminal p $6^{*}$ 
residues at the LZ/PR junction were created and designated DWz/PR and DWz//PR, respectively. Their LZ mutant counterparts were designated DKz/PR and DKz//PR, respectively (Fig. 2A).

Western immunoblot analysis data indicate that, in contrast to DWzPR, DKzPR produced substantial amounts of Pr55gag and p41gag, with barely detectable virusassociated p24gag (Fig. 2B, lane 7). Studies suggest that mature PR mediates virus maturation to yield p24gag, an indicator of virus particle processing efficiency. In contrast, PR precursor (p6*-PR), which possesses partial enzymatic activity, is incapable of efficiently processing all cleavage sites to yield mature virus-associated p24gag (18, 35). Insufficient virus particle processing with low levels of mature p24gag indicates, at least in part, a defect in PR maturation. Accordingly, replacing $\mathrm{p} 6^{*}$ with the mutant LZ may have interfered with the PR maturation process (see below). Surprisingly, the presence of the last four or six C-terminal $\mathrm{p} 6^{*}$ residues directly adjacent to the PR N terminus restored DWzPR virus yields to near-wt levels. We found that DWz//PR released virus particles at a relatively lower efficiency than other assembly-competent mutants (Fig. 2C). This may be due in part to the enhancement of Pr55gag cleavage prior to Pr55gag assembly into virus particles. In addition, all LZ replacement constructs with as few as four remaining C-terminal $p 6^{*}$ residues displayed virus particle processing profiles similar to that of the wt or Dp6*PR, regardless of the insertion of a mutant LZ (Fig. 2B, lanes 5 and 6 and lanes 8 and 9). Data from the quantification of virus particle processing efficiency indicate that DWz/PR and DKz/PR exhibited lower virus particle processing efficiency than DWz//PR and DKz//PR compared to the wt (Fig. 2D). This suggests that the presence of additional C-terminal $6^{*}$ residues can help PR activation. Combined, these data suggest that the C-terminal p6* tetrapeptide substantially mitigated the impacts of inserted foreign proteins on PR activation.

LZ replacement mutants produce infectious virions. Since release from a PR precursor is essential for full PR function, anti-HIV-1 PR serum was used to probe virions. As expected, the processing-defective DKzPR contained barely detectable PR and only partially processed PR-associated products (Fig. 2E, lane 7), suggesting a defect in PR activation. In contrast, the wt and all processing-competent mutants contained mature and readily detected PR. Note that since the constructs contained two PR domains, the bands corresponding to mature PR may represent either active or inactive PR. Migrating bands at positions corresponding to $6^{*}$-PR or LZ-PR became noticeable with longer Western blot exposures (Fig. 2E, lower panel). Bands that migrated at a slightly lower rate than mature PR were also observed in the LZ replacement mutants, possibly due to nonspecific degraded PR precursors.

Since processing-competent mutant p66/51RT was also detectable (Fig. 2E, top panel), we ran tests to determine whether these mutants were capable of producing infectious virions when pseudotyped with vesicular stomatitis virus glycoprotein (VSV$G)$. As predicted, no infectious virions were detected for the assembly-defective DWzPR or processing-defective DKzPR (Table 1). Despite containing substantial amounts of RT and displaying a wt virus particle processing profile, Dp6*PR exhibited less than one-third the infectivity of the wt. A possible explanation is that the inactivated PR copy might interfere with normal PR function, thereby disrupting the virus particle maturation process - a result that Western blot analysis cannot detect. This would contribute, at least in part, to reduced virus infectivity. Although DWz/PR, DWz//PR, DKz/PR, and DKz//PR mediated Gag processing in a manner similar to that of the wt (Fig. 2B), they exhibited infectivity at levels less than $3 \%$ of that of the wt. In addition to interference from the inactivated PR copy, deficient viral enzyme incorporation and insufficient virus particle maturation largely accounted for the poor infectivity.

Removal of PR downstream sequence does not diminish the leucine zipperinduced enhancement effect on Gag cleavage. RT and IN removal significantly affects Gag processing efficiency (36). We therefore reasoned that decreasing Gag cleavage efficiency by removing the downstream PR sequence might trigger DWzPR Gag release prior to PR cleavage. To test this hypothesis, a stop codon was inserted at 
A WT

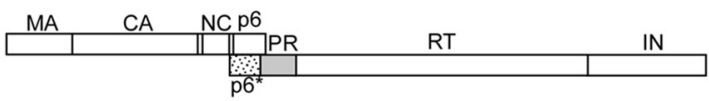

Dp6*PR

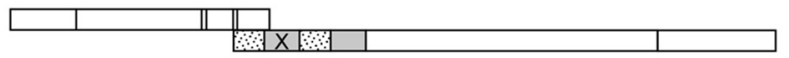

DWzPR

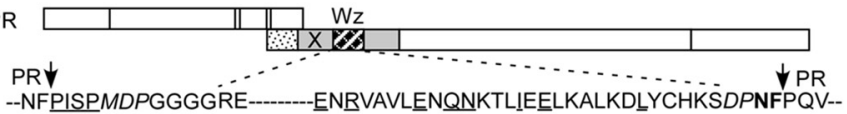

DWz/PR
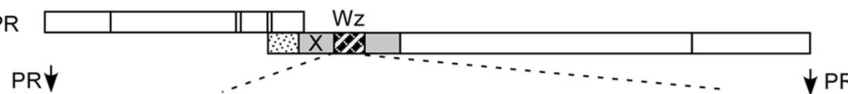

-NFPISPMDPGGGGRE---ENRVAVLENQNKTLIEELKALKDLYCHKS̈DPSFNFPQV-

DWZI/PR

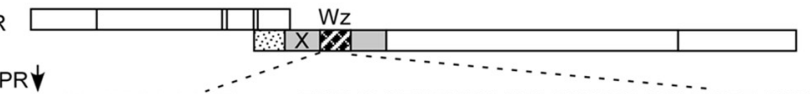

-NFPISPMDPGGGGRE-
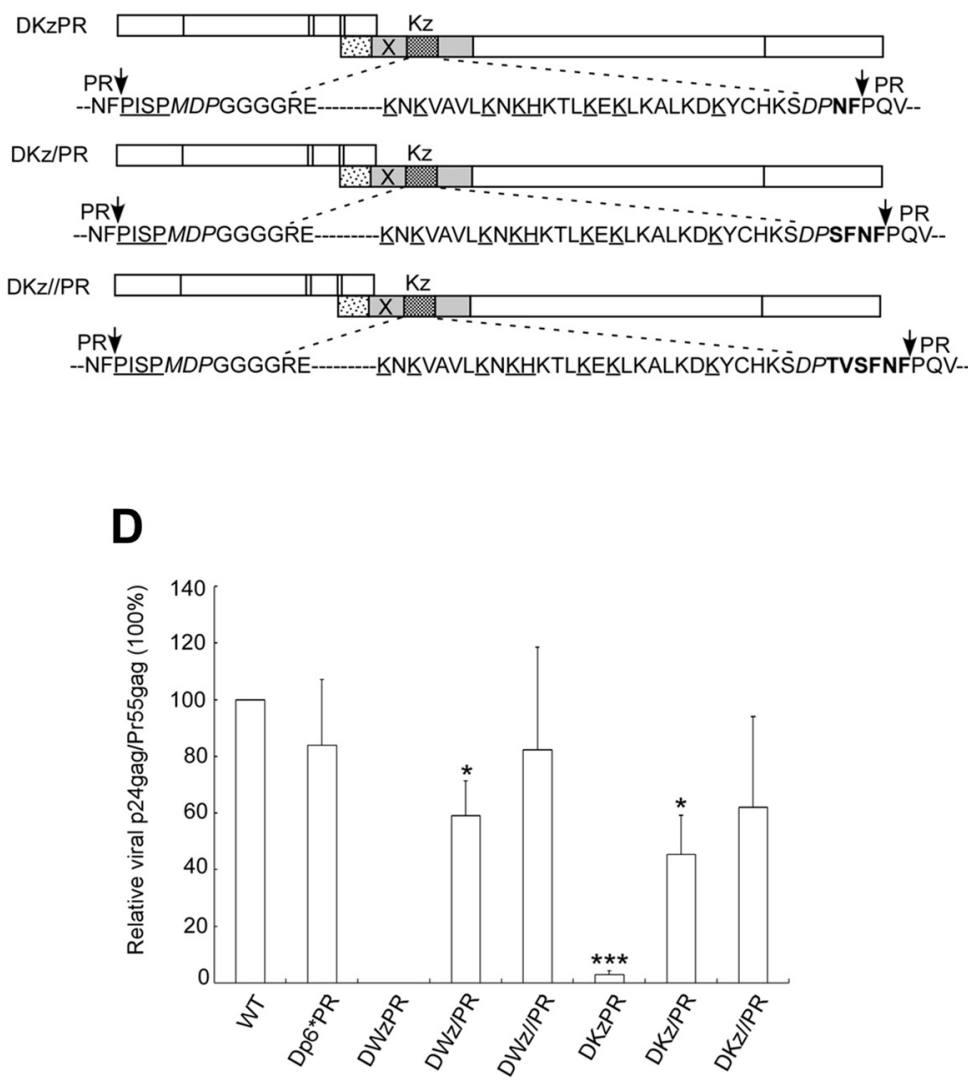

$\mathbf{E}$

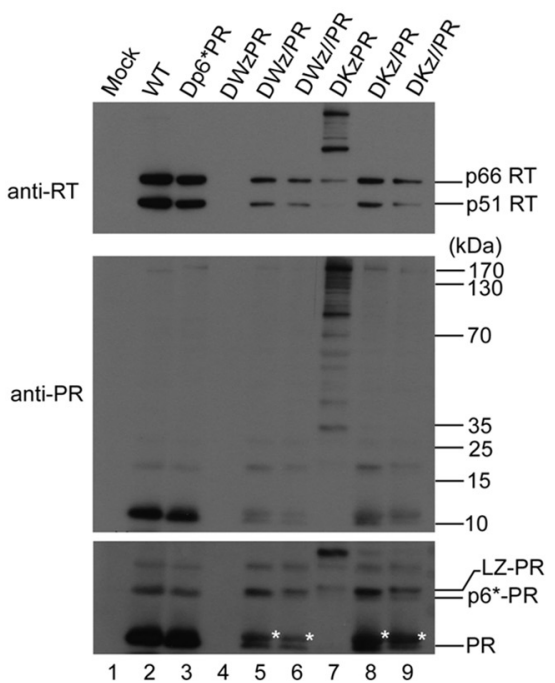

FIG 2 The presence of four C-terminal p6* residues counteracts the leucine zipper enhancement effect on Gag processing. (A) Schematic representations of HIV-1 Gag and Gag-Pol expression constructs. HIV-1 Gag protein domains and pol-encoded proteins are indicated as described in the Fig. 1 legend. Indicated are amino acid residues in the junction area. The "Wz" and "Kz" boxes denote wt and mutant leucine zipper domains, respectively. The mutant LZ contained Lys or His residue replacements of the corresponding wt amino acid residues (underlined). The two, four, or six C-terminal p6* residues remaining at the LZ/PR junction are shown in boldface. The underlined "PISP" indicates remaining N-terminal RT residues. Altered or additional residues are in italic. (B) $293 \mathrm{~T}$ cells were transfected with the designated constructs. At 48 to $72 \mathrm{~h}$ posttransfection, culture supernatants and cells were collected and subjected to Western immunoblotting. (C) Relative virus assembly efficiencies of HIV-1 mutants. Gag proteins from medium or cell samples were quantified by scanning mutant and wt p24gag-associated band densities from immunoblots. Ratios of total Gag protein levels in the media to those in cells were determined for each construct and compared with wt release levels by dividing the release ratio for each mutant by the wt ratio in parallel experiments. Error bars indicate standard deviations. ${ }^{*}, P<0.05$. (D) Relative virus particle processing efficiencies of HIV-1 mutants. Virus-associated Pr55gag and p24gag levels were quantified by scanning immunoblot band densities. Ratios of p24gag to p55gag were determined for each mutant and normalized to those of the wt in parallel experiments. Error bars indicate standard deviations. ${ }^{*}, P<0.05 ;{ }^{* *}, P<0.001$. (E) To detect PR-associated products, aliquots of supernatant samples from panel B were separated on 4 to $12 \%$ bis-Tris gradient gels. Membrane-bound proteins were first probed with anti-PR serum, stripped, probed with anti-RT serum, and then probed with anti-p24CA and anti-p17MA monoclonal antibodies. Longer exposure produced the blot at the bottom. Asterisks denote incompletely processed or degraded PR-associated products. The positions of molecular mass standards (in kDa) are indicated on the right (middle panel). 
TABLE 1 Infectivities of HIV-1 mutants

\begin{tabular}{|c|c|c|c|c|}
\hline \multirow[b]{2}{*}{ Construct $^{a}$} & \multicolumn{2}{|c|}{ Titer (CFU/ml) } & \multirow{2}{*}{$\begin{array}{l}\text { Infectivityc } \\
(\%)\end{array}$} & \multirow{2}{*}{$\begin{array}{l}\text { Mean infectivity } \\
(\%) \pm \text { SD }\end{array}$} \\
\hline & Mutant & Wild type $^{b}$ & & \\
\hline \multirow[t]{3}{*}{ Dp6*PR } & 47,200 & 255,000 & 19 & \\
\hline & 81,200 & 250,000 & 32 & \\
\hline & 76,000 & 280,000 & 27 & $26.0 \pm 6.5$ \\
\hline \multirow[t]{3}{*}{ DWzPR } & 0 & 255,000 & 0 & \\
\hline & 0 & 250,000 & 0 & \\
\hline & 0 & 280,000 & 0 & 0 \\
\hline \multirow[t]{3}{*}{$\mathrm{DWz} / \mathrm{PR}$} & 5,210 & 255,000 & 2 & \\
\hline & 6,750 & 250,000 & 3 & \\
\hline & 5,700 & 280,000 & 2 & $2.3 \pm 0.6$ \\
\hline \multirow[t]{3}{*}{ DWz//PR } & 3,920 & 255,000 & 2 & \\
\hline & 7,850 & 250,000 & 3 & \\
\hline & 6,500 & 280,000 & 2 & $2.3 \pm 0.6$ \\
\hline \multirow[t]{3}{*}{ DKzPR } & 0 & 255,000 & 0 & \\
\hline & 0 & 250,000 & 0 & \\
\hline & 0 & 280,000 & 0 & 0 \\
\hline \multirow[t]{3}{*}{$\mathrm{DKz} / \mathrm{PR}$} & 2,980 & 255,000 & 1 & \\
\hline & 2,820 & 250,000 & 3 & \\
\hline & 2,880 & 280,000 & 1 & $1.7 \pm 1.2$ \\
\hline \multirow[t]{3}{*}{ DKz//PR } & 1,890 & 255,000 & 0.7 & \\
\hline & 1,640 & 250,000 & 0.6 & \\
\hline & 1,920 & 280,000 & 0.7 & $0.7 \pm 0.1$ \\
\hline
\end{tabular}

${ }^{a}$ Constructs were cotransfected with VSV-G expression vectors into $293 \mathrm{~T}$ cells. At $48 \mathrm{~h}$ posttransfection, supernatants were collected, filtered, and used to infect HeLa cells. Infection and selection of drug-resistant colonies were performed as described in Materials and Methods.

${ }^{b}$ Wild-type (HIVgpt) titers were determined in parallel experiments performed in triplicate.

cRatios of viral titers to Gag protein levels (obtained via immunoblot band density quantification) were

determined for each mutant and normalized to those of the wt in parallel experiments.

the PR C terminus of DWzPR. The wt and Gag assembly- and processing-competent Dp6*PR and DWz/PR served as controls (Fig. 3A). As expected, all three exhibited significant increases in virus-associated Pr55gag and p41 following RT and IN removal, suggesting reduced Gag processing efficiency (Fig. 3B, upper middle panel, lanes 3, 5, and 9). However, medium-associated Gag was still barely detectable, indicating that RT and IN deletion did not significantly reduce DWzPR Gag processing efficiency (Fig. 3B, lanes 6 and 7). According to these data, LZ replacement of $p 6^{*}$ efficiently triggered premature PR activation independent of the PR downstream sequence.

Four amino acids on the p6* C-terminal side are important for PR activation. The above-described results support the idea that the four C-terminal $p 6^{*}$ residues are important for regulating PR activation. In a previous study, we found that Dp6*PR with a $6^{*}$ deletion was defective in virus processing. This defect likely resulted from inefficient PR activation, since only two C-terminal $p 6^{*}$ residues remained at the PR $\mathrm{N}$-terminal end. To test this possibility, we constructed D/PR and D//PR, which had four and six C-terminal $p 6^{*}$ residues remaining at the $\mathrm{p} 6^{*} / \mathrm{PR}$ junction, respectively (Fig. $4 \mathrm{~A}$ ). To determine whether NC is capable of facilitating PR activation when inserted into the deleted p6* region, three additional constructs (DNCPR, DNC/PR, and DNC//PR) were created by replacing $\mathrm{p}^{*}$ with $\mathrm{NC}$, which possesses a self-association characteristic (37). Our data indicate that the virus-associated Gag and Gag-Pol products of DPR and DNCPR were largely in precursor form, suggesting a severe defect in virus particle processing (Fig. 4B). In contrast, D/PR, D//PR, DNC/PR, and DNC//PR all contained readily detected $\mathrm{p} 24 \mathrm{gag}$ and $\mathrm{p} 17 \mathrm{gag}$, thereby confirming the importance of the four C-terminal $\mathrm{p}^{*}$ residues for modulating PR activation.

Both $\mathrm{D} / \mathrm{PR}$ and $\mathrm{D} / / \mathrm{PR}$ contained significant levels of RT and readily detectable mature PR (Fig. 4B, lanes 5 and 6). However, single-cycle infection assay results showed 
A
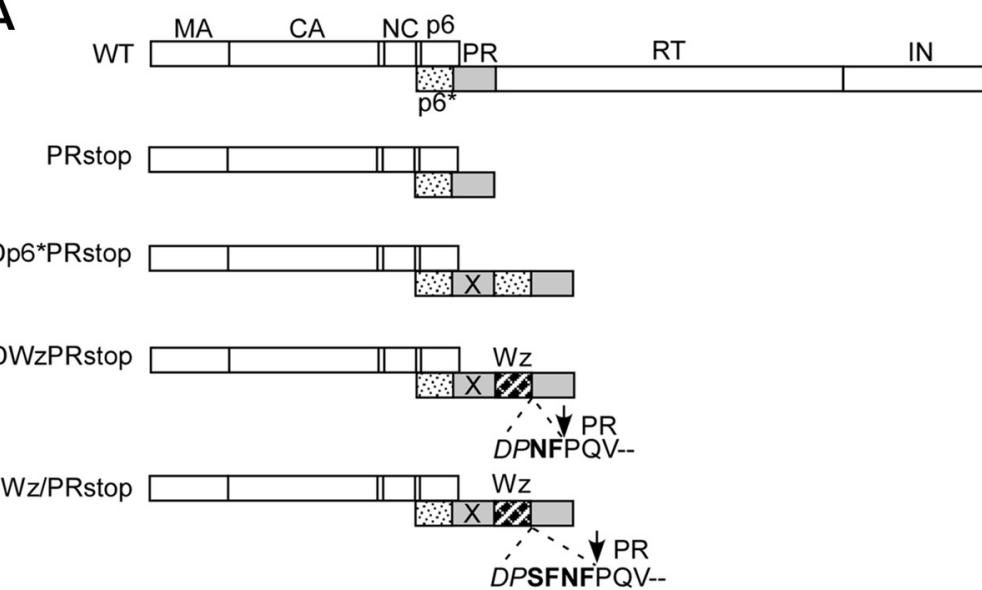

B

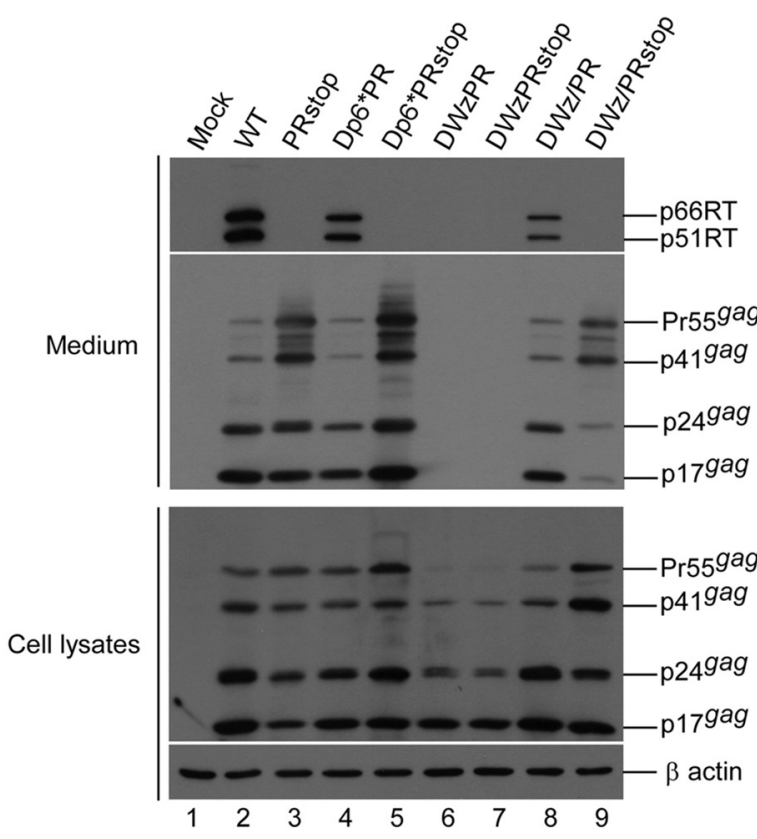

FIG 3 Leucine zipper-induced Gag cleavage enhancement is independent of the downstream PR sequence. (A) Schematic representations of HIV-1 Gag and Gag-PR expression constructs. PRstop contained a stop codon insertion at the PR-RT junction (codon sequence, 5'TTTCCCATTAGCCCTTAG-3'); RT codons are underlined. Recombining PRstop with Dp6*PR, DWzPR, or DWz/PR (shown in Fig. 2A) yielded Dp6*PRstop, DWzPRstop, or DWz/PRstop. Remaining C-terminal $6^{*}$ residues at the LZ/PR junction are shown in boldface. (B) 293T cells were transfected with the designated constructs. At $48 \mathrm{~h}$ posttransfection, cells and supernatants were collected and subjected to Western immunoblotting.

that they also exhibited infectivity levels of between $2 \%$ and $3 \%$ of that of the wt (data not shown). Further, D/PR and D//PR exhibited significantly lower virus maturation efficiencies than the wt (Fig. 4C), with both containing substantial amounts of immature PR (Fig. 4B, asterisks). The inactivated PR copy may have interfered with the PR function. These scenarios may account for their low infectivity, at least in part. In the cases of DNC/PR and DNC//PR, virus-associated RT was barely detectable. Under overexposure conditions, detected PR from DNC/PR and DNC//PR was largely in precursor form (Fig. $4 \mathrm{~B}$, bottom panel, lanes 8 and 9), which is consistent with their virus particle processing defects (Fig. 4C). As expected, infectivity titers for both were very low, approximately $0.1 \%$ of the wt level (data not shown). These data suggest that NC, when substituted for $\mathrm{p}^{*}$, did not facilitate PR dimer interaction, despite possessing a self-association capacity to promote Gag multimerization $(37,38)$. NC in the Gag domain of DNCPR Gag-Pol might interact intra- or cross-molecularly with NC in the deleted $p 6^{*}$ region, 
A

WT

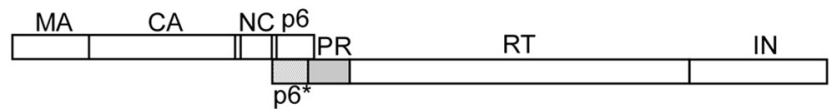

Dp6*PR

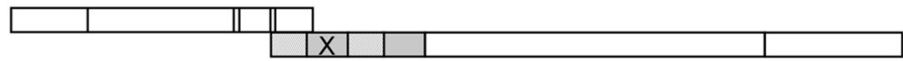

DPR

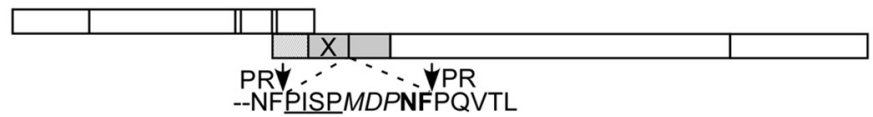

D/PR

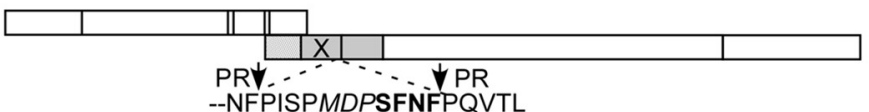

D//PR

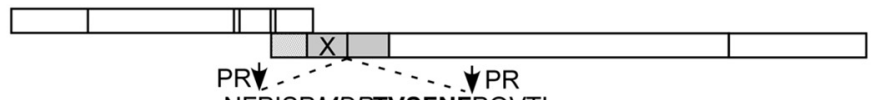

-NFPISPMDPTVSFNFPQVTL

DNCPR

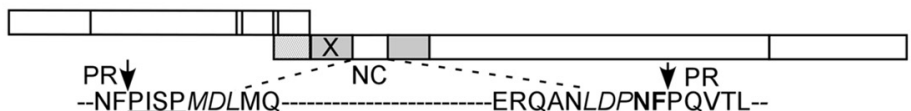

DNC/PR

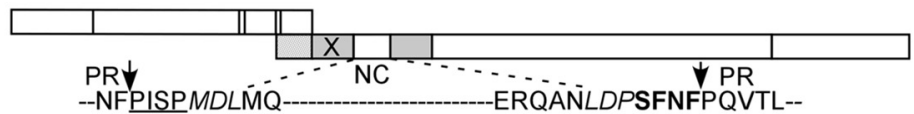

DNC//PR
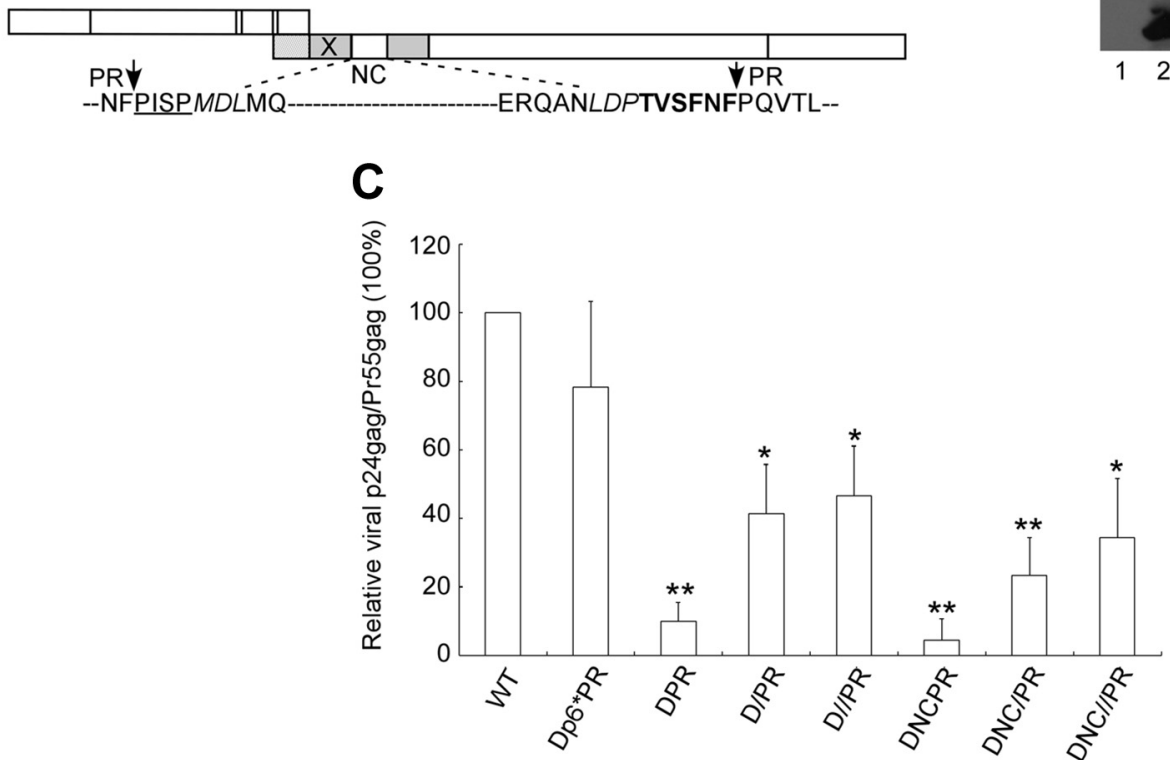

FIG 4 Importance of C-terminal p6* tetrapeptide to PR-mediated virus processing. (A) Schematic representations of HIV-1 Gag and Gag-Pol expression constructs. HIV-1 Gag protein domains and pol-encoded proteins are indicated as described in the Fig. 1 legend. Amino acid residues in the junction area are also indicated. Remaining C-terminal p6* residues are shown in boldface. The underlined "PISP" indicates remaining N-terminal RT residues. Altered or foreign amino acid residues are shown in italic. (B) 293T cells were transfected with the designated constructs. At 48 to $72 \mathrm{~h}$ posttransfection, supernatants were collected, filtered, and centrifuged through $20 \%$ sucrose cushions. Samples were separated on 4 to $12 \%$ bis-Tris gradient gels. HIV-1 PR, RT, and Gag were sequentially probed with antisera as described in the Fig. 2B legend. Asterisks indicate incompletely processed PR precursor likely representing PR linked with amino acids (PISPMDPSFNF or PISPMDPTVSFNF) at the $\mathrm{N}$ terminus. The positions of molecular mass standards (in $\mathrm{kDa}$ ) are indicated on the right (lower middle panel). Longer exposure produced the bottom panel blot for viewing PR-associated products. (C) Relative virus particle processing efficiencies of HIV-1 mutants. Virus-associated Pr55gag and p24gag levels were quantified by scanning immunoblot band densities. Ratios of p24gag to p55gag were determined for each mutant and normalized to those of the wt in parallel experiments. Error bars indicate standard deviations. ${ }^{*}, P<0.05 ;{ }^{* *}, P<0.01$.

resulting in a PR activation defect due to a disturbance of Gag-Pol or PR dimer interface interactions. This may explain why (i) DWzPR enhanced Gag cleavage but DNCPR did not and (ii) compared to the wt, DNC/PR and DNC//PR exhibited relatively lower virus particle processing efficiencies than their LZ replacement counterparts DWz/PR and DWz//PR (Fig. 2D versus 4C). 
A
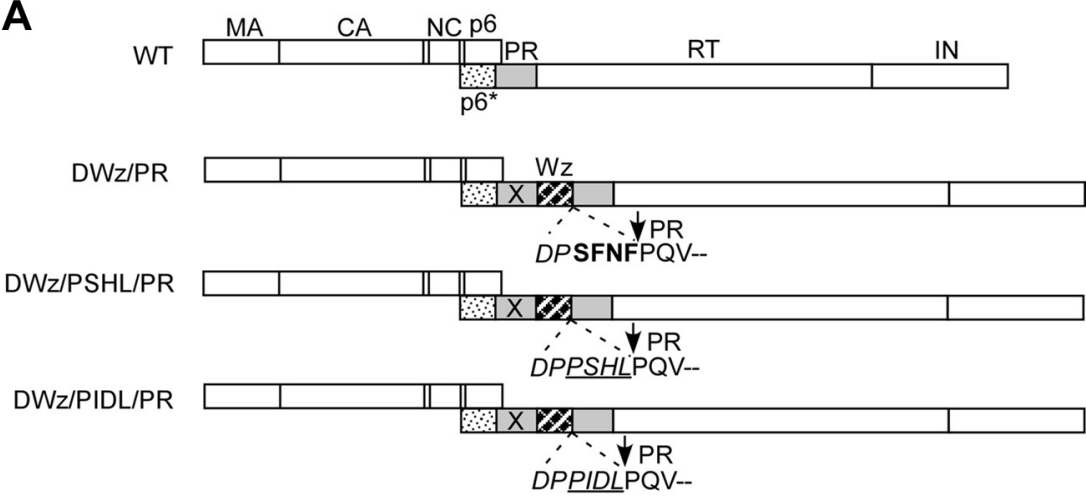

B
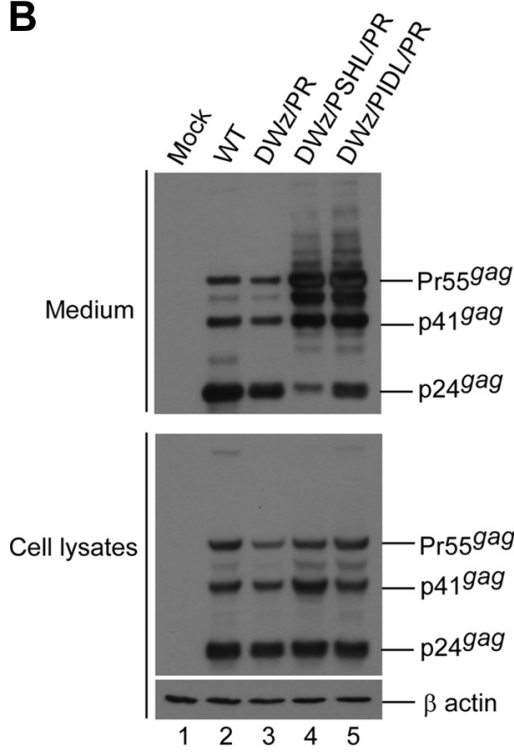

C

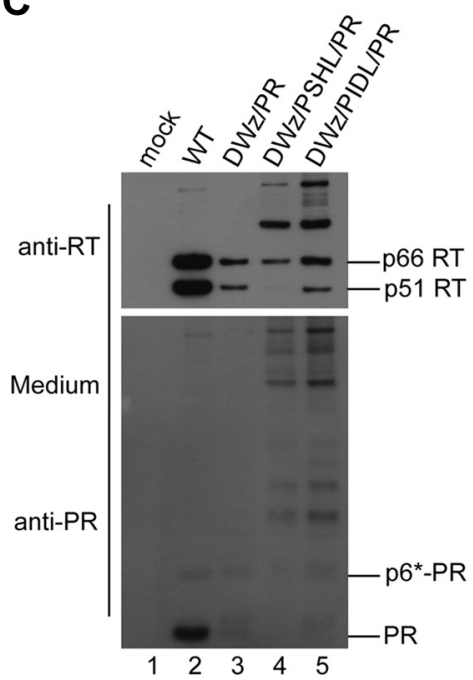

FIG 5 C-terminal p6* tetrapeptide mutations mitigate the leucine zipper-induced Gag cleavage enhancement. (A) Schematic representations of HIV-1 Gag and Gag-Pol expression constructs. HIV-1 Gag protein domains, pol-encoded proteins, and amino acids in the junction area are indicated as described in the Fig. 2 legend. The boldface "SFNF" indicates the wt C-terminal p6* tetrapeptide, while the underlined amino acid residues denote substitutions for the wt C-terminal p6* tetrapeptide. Altered or foreign amino acid residues are shown in italic. (B and C) 293T cells were transfected with the designated constructs. At 48 to $72 \mathrm{~h}$ posttransfection, supernatants were collected, filtered, and centrifuged through $20 \%$ sucrose cushions. Samples were separated on 4 to $12 \%$ bis-Tris gradient gels. Membrane-bound proteins were first probed with anti-PR serum, stripped, probed with anti-RT serum $(C)$, and probed with anti-p24CA monoclonal antibody (B).

The C-terminal $p 6^{*}$ tetrapeptide is nonspecific when mitigating LZ-induced Gag cleavage enhancement. The results shown in Fig. 2 suggest that the C-terminal p $6^{*}$ tetrapeptide counteracted the LZ-incurred assembly defect. In order to confirm that other amino acids, when substituted for the C-terminal p6* tetrapeptide SFNF, are also capable of reducing LZ-induced Gag cleavage enhancement, we replaced SFNF with either PSHL or PIDL (two tetrapeptide substitution mutants) (Fig. 5A). According to one previous report, PSHL or PIDL substitutions for SFNF significantly affected PR activation and/or impeded PR maturation (18). Further analysis indicates that both tetrapeptide substitution mutants were capable of producing substantial amounts of virusassociated Gag; however, in contrast to DWz/PR, the two tetrapeptide substitution mutant particles were largely unprocessed or incompletely processed (Fig. 5B, upper panel, lanes 3 to 5). The two substitution mutants also exhibited insufficient Gag-Pol processing, with mature PR barely detectable compared to its precursor forms (Fig. 5C, lanes 4 and 5). These results suggest that foreign amino acid substitutions for the 
C-terminal $p 6^{*}$ tetrapeptide markedly affected PR activation, despite their characteristic of counteracting LZ-incurred assembly defects.

\section{DISCUSSION}

The assertion that $\mathrm{p} 6^{*}$ prevents early PR activation is based largely on in vitro analyses of PR precursor (PR containing amino-terminally extended $\mathrm{p} 6^{*}$ residues) (31-34). The mitigating effect of the $p 6^{*}$ domain on PR maturation, if any, has not been shown in a virus assembly context. We found that the presence of four C-terminal p6* residues markedly impeded enhanced Gag cleavage via a leucine zipper insertion in deleted $\mathrm{p}^{*}$ regions, suggesting that C-terminal $\mathrm{p}^{*}$ residues can prevent early PR activation.

All $6^{*}$ substitution mutants were derived from Dp6*PR, which exhibited a virus assembly and processing profile similar to that of the wt. Dp6*PR contains virusassociated PR and RT at levels comparable to those of the wt and, according to single-cycle infection assay results, displays infectivity at a level approximately $30 \%$ of that of the wt. These data suggest that the Dp6*PR construct exerts no major impacts on Gag and Gag-Pol expression and that Gag-Pol is capable of undergoing autoprocessing to produce a functional PR that mediates virus maturation. It is likely that inactivated upstream PR interferes with normal PR function, which may partly account for the reduced Dp6*PR infectivity we observed. Negative effects from inactivated PR, if any, would reduce the remarkable Gag processing increase observed for DWzPR. PR contains several sites that are sensitive to PR autoproteolysis, and it therefore tends to undergo rapid self-degradation $(39,40)$. This may partly explain why intracellular PR was barely detectable.

p6* contributes little to wt PR dimerization or enzyme activity, although it does facilitate the dimerization of a drug-resistant PR variant and has been shown to restore its enzymatic activity in a bacterial assay system (41). Apparently the Gag-Pol sequence outside the PR domain largely determines PR dimer interaction efficiency and folding in order to trigger PR autoprocessing $(23,31,33,36,42-45)$. Data from an uncoupled Gag and Gag-Pol expression system indicate that the removal of most of the central p ${ }^{*}$ domain or the insertion of a small foreign protein in the deleted region did not exert significant impacts on HIV-1 replication. However, the insertion of a large heterologous protein such as green fluorescent protein (GFP), with a molecular weight about five times that of $\mathrm{p} 6^{*}$, resulted in barely detectable mature p24gag, indicating a severe defect in virus maturation (46). This suggests that the inserted foreign protein disrupted the PR dimer folding that is required for proper maturation. The inserted LZ in the p6*-deleted region was approximately the same size as $p 6^{*}$, which may not have significantly affected PR precursor folding as long as the C-terminal p6* tetrapeptide was present.

All of the LZ replacement constructs exhibited similar virus assembly and processing profiles when the last four C-terminal $\mathrm{p} 6^{*}$ residues remained at the $\mathrm{p} 6^{*} / \mathrm{PR}$ junction. We found that DWzPR and DKzPR both contained the last two C-terminal p6* residues, but the former exhibited remarkably enhanced Gag cleavage and the latter displayed a severe Gag cleavage defect (Fig. 2). These data suggest that the four C-terminal p6* residues are functionally involved in preventing premature PR activation in addition to being required for PR maturation. It is possible that at some point the SFNF C-terminal p6* tetrapeptide within DWz/PR and DWz//PR may have exerted spacer effects that prevented the inserted LZ from facilitating PR dimer interactions. We observed that virus particles produced by DWz/PR were markedly defective in processing when the SFNF tetrapeptide was replaced with foreign amino acid residues (Fig. 5). This suggests that the four foreign residues significantly affected PR activation, despite their influence in terms of mitigating the LZ replacement effect on PR activation when placed between the PR and inserted LZ. Defects in virus particle processing were noted when Ser-Phe residues in both DPR and DNCPR were replaced by Asp-Pro residues (Fig. 4). These results agree with previously reported findings that mutation substitutions at the C-terminal $p 6^{*}$ tetrapeptide strongly interfere with PR release and virus maturation (18). 
Thus, it is likely that the ability of PSHL and PIDL substitutions to mitigate LZ-induced Gag cleavage enhancement is due, at least in part, to markedly reduced PR activation. Combined, our data suggest that the C-terminal $p 6^{*}$ tetrapeptide plays a role in the spatiotemporal regulation of PR maturation.

Analyses of PR precursor structure and function suggest that p6*-PR is relatively unstable and possesses low enzymatic activity (33,47-49). The lack of stability may allow PR precursor to adopt flexible orientations for the transient formation of dimer complexes involving the mature PR dimer interface. One PR miniprecursor (SFNFPR, containing as few as four C-terminal p6* residues) has been described as being capable of mimicking a full-length PR precursor model (49). According to that model, transient encounter complex dimers display more flexible orientations than mature PR, although at very low levels (approximately 3 to $5 \%$ of population in solution); this may account for the very low PR precursor enzymatic activity levels we observed. However, transient encounter complex dimers are capable of adopting transient insertions of $\mathrm{N}$-terminal extensions (including the $\mathrm{p} 6^{*} / \mathrm{PR}$ cleavage site) in substrate clefts to facilitate cleavage at the PR N terminus (i.e., intramolecular cleavage), resulting in the formation of a stable and mature PR dimer $(43,49)$. Accordingly, it is likely that the presence of as few as four C-terminal $\mathrm{p}^{*}$ residues would allow for PR folding and maturation in a precise spatiotemporal manner so as to block premature PR activation triggered by a potent upstream dimerization motif, as observed with DWzPR. Since it lacks an intact C-terminal p6* tetrapeptide, LZ within DWzPR may dictate PR maturation by stabilizing $\mathrm{PR}$ precursor dimer structure and/or facilitating PR dimer formation, resulting in premature PR activation.

In conclusion, we found that the replacement of $\mathrm{p} 6^{*}$ with a leucine zipper (LZ) dimerization motif eliminated virion production due to the significant enhancement of PR-mediated Gag cleavage efficiency. As few as four remaining C-terminal $p 6^{*}$ residues at the $6^{*} / \mathrm{PR}$ junction were sufficient for counteracting the LZ-incurred defect in virus assembly, which partly supports the idea of PR function mediating virus maturation. Our results provide evidence in support of the idea that C-terminal $\mathrm{p} 6^{*}$ residues contribute to virus assembly and maturation by both modulating and preventing early PR activation, and they encourage further research involving the proposal that interfering with PR maturation by targeting PR precursor may represent an alternative approach for anti-HIV therapies.

\section{MATERIALS AND METHODS}

Plasmid construction. The HIV-1 proviral plasmid HIVgpt is considered the backbone of all expression constructs (50). The constructs used in this research were mostly derived from Dp6*PR and DPR. As previously described (24), Dp6*PR contains a p6* domain between an inactivated and an active PR. In contrast, DPR contains BamHI-linked duplicate PR pairs with an inactivated proximal PR. To insert a leucine zipper (LZ) between the wt and inactivated PR domains, we subcloned DPR mutations into a pBRCla-Sal plasmid cassette containing an HIV-1 coding sequence from Clal (nucleotide [nt] 831) to Sall (nt 5786). The resulting construct was designated pBRClaSal/DPR. PRWzPR and PRKzPR, both of which contain a BamHI-flanking wt (Wz) or mutant (Kz) human CREB LZ between the PR pairs (24), were digested with BamHI and ligated into pBRClaSal/DPR, respectively, yielding DWzPR and DKzPR constructs. Mutant LZ- and wt-containing plasmids for constructing PRWzPR and PRKzPR were gifts from E.

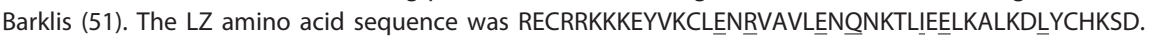
The underlined amino acid residues were mutated to Lys or His (Fig. 2), thus yielding the mutant LZ (52).

The following BamHI-containing primers (forward) were used to amplify PR precursor-coding fragments containing four (SFNF) or six (TVSFNF) C-terminal p6* residues or C-terminal p6* tetrapeptide mutations (PSHL or PIDL): SFNF, 5'-CGGGATCCTTCCTTTAACTTCCCTCAGGTCACGTTATGG-3'; TVSFNF, 5'CGGGATCCTACTGTATCCTITAACTTCCCTCAGGTC-3'; PSHL, 5'-CGGGATCCTCCCTCTCACCTCCCTCAGGTCA CTCTITGG-3'; PIDL, 5'-CGGGATCCTCCCATTGACCTCCCTCAGGTCACTCTITGG-3'. DPR served as a template. The reverse primer (nt 3116 to 90 ) sequence was 5'-TACATACAAATCATCCATGTTATTGATA-3'. Each PCR-amplified fragment was purified, digested with BamHI and EcoRV, and subcloned into the pBRClaSal/DPR cassette. Next, BamHI-flanking wt (Wz) and mutant (Kz) LZ fragments derived from PRWzPR and PRKzPR were inserted into each pBRClaSal/DPR recombinant with either four or six $C$-terminal $p 6^{*}$ residues remaining at the $P R N$ terminus. The yields were designated $D W z / P R$, DWz//PR, DKz/PR, and DKz//PR. Mutated DPR recombined with DWz/PR and DWz//PR yielded D/PR and $D / / P R$, respectively. 
To clone DNCPR, fragments containing NC-coding sequences were PCR amplified with a 5'-AATTC AGCTACAGATCTGATGCAG-3' forward primer and 5'-GATCTTCGGATCCAAATTAGCCTG-3' reverse primer. Amplified fragments were digested with Bglll and BamHI and cloned into pBRClaSal/DPR that had been digested with BamHI. DNC/PR and DNC//PR were, derived by recombining the DNCPR mutation with $D / P R$ and $D / / P R$, respectively.

Cell culture, transfection, and infection. 293T cells and HeLa cells were maintained in Dulbecco's modified Eagle medium (DMEM) supplemented with $10 \%$ fetal calf serum. At 16 to $24 \mathrm{~h}$ prior to transfection, confluent 293T cells were trypsinized, split 1:10, and seeded onto 10-cm dish plates. Twenty micrograms of plasmid DNAs from each construct was used to transfect 293T cells by the calcium phosphate precipitation method, with the addition of $50 \mu \mathrm{M}$ chloroquine to enhance transfection efficiency.

For a single-cycle infection assay, $20 \mu \mathrm{g}$ of wt or mutant HIVgpt was cotransfected with $5 \mu \mathrm{g}$ of the VSV-G protein expression vector pHCMV-G (53). Virus-containing supernatant was collected and filtered, and aliquots of the filtrate were diluted to infect HeLa cells in the presence of $4 \mu \mathrm{g} / \mathrm{ml}$ Polybrene. The remaining supernatant and cell samples were prepared and subjected to Western blotting. Twenty-four hours after infection, cells were trypsinized, split into dishes, and refed with medium containing drug selection cocktail (54). Drug-resistant colonies were fixed and stained with $50 \%$ methanol containing $0.5 \%$ methylene blue. Numbers of drug-resistant colonies were converted into titers (CFU/ml). Infectivity was expressed as the ratio of the mutant titer to the titer of the wt, and normalized to Gag protein levels in parallel experiments.

Western immunoblot analysis. Supernatants from transfected 293T cells were filtered through $0.45-\mu \mathrm{m}$-pore-size filters, followed by centrifugation through $2 \mathrm{ml}$ of $20 \%$ sucrose in TSE (10 mM Tris-HCl [pH 7.5], $100 \mathrm{mM} \mathrm{NaCl}, 1 \mathrm{mM}$ EDTA) buffer containing $0.1 \mathrm{mM}$ phenylmethylsulfonyl fluoride (PMSF) at $4^{\circ} \mathrm{C}$ for $40 \mathrm{~min}$ at $274,000 \times g$ (SW41 rotor at 40,000 rpm). The viral pellets were then suspended in IPB buffer ( $20 \mathrm{mM}$ Tris-HCl, pH 7.5, $150 \mathrm{mM} \mathrm{NaCl}, 1 \mathrm{mM}$ EDTA, $0.1 \%$ SDS, $0.5 \%$ sodium deoxycholate, $1 \%$ Triton X-100, $0.02 \%$ sodium azide) containing $0.1 \mathrm{mM}$ phenylmethylsulfonyl fluoride (PMSF). The cells were rinsed with ice-cold phosphate-buffered saline (PBS), collected in $1 \mathrm{ml}$ PBS, and pelleted at 2,500 rpm for $5 \mathrm{~min}$. The cell pellets were resuspended in $250 \mu \mathrm{l}$ of IPB containing $0.1 \mathrm{mM}$ PMSF and then subjected to microcentrifugation at $4^{\circ} \mathrm{C}$ for $15 \mathrm{~min}$ at $13,700 \times g$ to remove cell debris. The supernatant and cell samples were mixed with equal volumes of $2 \times$ sample buffer $(12.5 \mathrm{mM}$ Tris-HCl [pH 6.8], $2 \%$ SDS, $20 \%$ glycerol, $0.25 \%$ bromophenol blue) containing $\beta$-mercaptoethanol (5\%), and boiled for $5 \mathrm{~min}$. Samples were subjected to $10 \%$ SDS-PAGE on 4 to $12 \%$ bis-Tris gradient gels (NuPage bis-Tris minigels; Thermo Fisher Scientific) and electroblotted onto nitrocellulose membranes. HIV-1 Gag/Gag-Pol were probed with anti-p24gag (mouse hybridoma clone 183-H12-5C) and/or anti-p17gag (American Type Culture Collection HB-8975) monoclonal antibodies capable of detecting mature p17gag. Membranebound HIV-1 PR was detected with a sheep antiserum. The primary antibody for HIV-1 RT was rabbit antiserum or a mouse anti-RT monoclonal antibody $(55,56)$. The secondary antibody was either a rabbit anti-mouse, donkey anti-rabbit, or anti-sheep horseradish peroxidase (HRP)-conjugated antibody (Jackson ImmunoResearch) at a dilution of 1:10,000 or 1:5,000. Protocols for detection of membrane-bound proteins were as described previously (57), using an enhanced chemiluminescence (ECL) detection system (SuperSignal West Pico chemiluminescent substrate; Thermo Fisher Scientific) according to the manufacturer's protocol.

Statistical analysis. Differences between control (wt) and experimental (mutant) groups were assessed using Student $t$ tests. Data are expressed as mean \pm standard deviation. Significance was defined as follows: ${ }^{*}, P<0.05 ;{ }^{* *} P<0.01$; and ${ }^{* * *}, P<0.001$.

\section{ACKNOWLEDGMENTS}

We thank the following individuals from the National Institutes of Health AIDS Research and Reference Reagent Program for their assistance in obtaining materials: Stephen Hughes (anti-RT monoclonal antibody MAb21) and D. Bailey and Mark Page (antiserum to HIV-1 PR).

\section{REFERENCES}

1. Swanstrom R, Wills J. 1997. Synthesis, assembly and processing of viral proteins, p 263-334. In Coffin J, Hughes S, Varmus H (ed), Retroviruses. Cold Spring Harbor Laboratory Press, Cold Spring Harbor, NY.

2. Lee S-K, Potempa M, Swanstrom R. 2012. The choreography of HIV-1 proteolytic processing and virion assembly. J Biol Chem 287: 40867-40874. https://doi.org/10.1074/jbc.R112.399444.

3. Pettit SC, Moody MD, Wehbie RS, Kaplan AH, Nantermet PV, Klein CA, Swanstrom R. 1994. The p2 domain of human immunodeficiency virus type $1 \mathrm{Gag}$ regulates sequential proteolytic processing and is required to produce fully infectious virions. J Virol 68:8017-8027.

4. Gottlinger HG, Sodroski JG, Haseltine WA. 1989. Role of capsid precursor processing and myristoylation in morphogenesis and infectivity of human immunodeficiency virus type 1. Proc Natl Acad Sci U S A 86: 5781-5785. https://doi.org/10.1073/pnas.86.15.5781.

5. Kohl NE, Emini EA, Schleif WA, Davis LJ, Heimbach JC, Dixon RA, Scolnick EM, Sigal IS. 1988. Active human immunodeficiency virus protease is required for viral infectivity. Proc Natl Acad Sci U S A 85:4686-4690. https://doi.org/10.1073/pnas.85.13.4686.

6. Peng C, Ho BK, Chang TW, Chang NT. 1989. Role of human immunodeficiency virus type 1-specific protease in core protein maturation and viral infectivity. J Virol 63:2550-2556.

7. Jacks T, Power M, Maslarz F, Luciw P, Barr P, Varmus H. 1988. Charac terization of ribosomal frame-shifting in HIV-1 gag/pol expression. Nature 331:280-283. https://doi.org/10.1038/331280a0.

8. Arrigo SJ, Huffman K. 1995. Potent inhibition of human immunodeficiency virus type 1 (HIV-1) replication by inducible expression of HIV-1 PR multimers. J Virol 69:5988-5994.

9. Hill M, Hooker C, Harrich D, Crowe S, Mak J. 2001. Gag-Pol supplied in trans is efficiently packaged and supports viral function in human immunodeficiency virus type 1. J Virol 75:6835-6840. https://doi.org/10 .1128/JVI.75.15.6835-6840.2001.

10. Krausslich H. 1991. Human immunodeficiency virus proteinase dimer as 
component of the viral polyprotein prevents particle assembly and viral infectivity. Proc Natl Acad Sci U S A 88:3213-3217. https://doi.org/10 .1073/pnas.88.8.3213.

11. Park J, Morrow C. 1991. Overexpression of the gag-pol precursor from human immunodeficiency virus type 1 proviral genomes results in efficient proteolytic processing in the absence of virion production. J Virol 65:5111-5117.

12. Rose JR, Babe LM, Craik CS. 1995. Defining the level of human immunodeficiency virus type 1 (HIV-1) protease activity required for HIV-1 particle maturation and infectivity. J Virol 69:2751-2758.

13. Shehu-Xhilaga M, Crowe SM, Mak J. 2001. Maintenance of the Gag/GagPol ratio is Important for human immunodeficiency virus type 1 RNA dimerization and viral infectivity. J Virol 75:1834-1841. https://doi.org/ 10.1128/JVI.75.4.1834-1841.2001.

14. Wang C-T, Chou Y-C, Chiang C-C. 2000. Assembly and processing of human immunodeficiency virus Gag mutants containing a partial replacement of the matrix domain by the viral protease domain. J Virol 74:3418-3422. https://doi.org/10.1128/JVI.74.7.3418-3422.2000.

15. Figueiredo A, Moore KL, Mak J, Sluis-Cremer N, de Bethune M-P, Tachedjian G. 2006. Potent nonnucleoside reverse transcriptase inhibitors target HIV-1 Gag-Pol. PLoS Pathog 2:e119. https://doi.org/10.1371/journal ppat.0020119.

16. Tachedjian G, Orlova M, Sarafianos SG, Arnold E, Goff SP. 2001. Nonnucleoside reverse transcriptase inhibitors are chemical enhancers of dimerization of the HIV type 1 reverse transcriptase. Proc Natl Acad Sci U S A 98:7188-7193. https://doi.org/10.1073/pnas.121055998.

17. Pan Y-Y, Wang S-M, Huang K-J, Chiang C-C, Wang C-T. 2012. Placement of leucine zipper motifs at the carboxyl terminus of HIV-1 protease significantly reduces virion production. PLoS One 7:e32845. https://doi .org/10.1371/journal.pone.0032845.

18. Ludwig C, Leiherer A, Wagner R. 2008. Importance of protease cleavage sites within and flanking human immunodeficiency virus type 1 transframe protein $6^{*}$ for spatiotemporal regulation of protease activation. J Virol 82:4573-4584. https://doi.org/10.1128/JVI.02353-07.

19. Partin K, Zybarth G, Ehrlich L, DeCrombrugghe M, Wimmer E, Carter C. 1991. Deletion of sequences upstream of the proteinase improves the proteolytic processing of human immunodeficiency virus type 1 . Proc Natl Acad Sci U S A 88:4776-4780. https://doi.org/10.1073/pnas.88.11 .4776 .

20. Tessmer U, Krausslich H-G. 1998. Cleavage of human immunodeficiency virus type 1 proteinase from the $\mathrm{N}$-terminally adjacent $\mathrm{p}^{*}$ protein is essential for efficient Gag polyprotein processing and viral infectivity. J Virol 72:3459-3463.

21. Paulus C, Ludwig C, Wagner R. 2004. Contribution of the Gag-Pol transframe domain $\mathrm{p}^{*}$ and its coding sequence to morphogenesis and replication of human immunodeficiency virus type 1. Virology 330: 271-283. https://doi.org/10.1016/j.virol.2004.09.013.

22. Paulus C, Hellebrand S, Tessmer U, Wolf H, Kräusslich H-G, Wagner R. 1999. Competitive inhibition of human immunodeficiency virus type-1 protease by the Gag-Pol transframe protein. J Biol Chem 274: 21539-21543. https://doi.org/10.1074/jbc.274.31.21539.

23. Louis J, Dyda F, Nashed N, Kimmel A, Davies D. 1998. Hydrophilic peptides derived from the transframe region of Gag-Pol inhibit the HIV-1 protease. Biochemistry 37:2105-2110. https://doi.org/10.1021/ bi972059x.

24. Yu F-H, Chou T-A, Liao W-H, Huang K-J, Wang C-T. 2015. Gag-Pol transframe domain $\mathrm{p}^{*}$ is essential for HIV-1 protease-mediated virus maturation. PLoS One 10:e0127974. https://doi.org/10.1371/journal .pone.0127974.

25. Bleiber G, Peters S, Martinez R, Cmarko D, Meylan P, Telenti A. 2004. The central region of human immunodeficiency virus type 1 p6 protein (Gag residues S14-I31) is dispensable for the virus in vitro. J Gen Virol 85: 921-927. https://doi.org/10.1099/vir.0.19576-0.

26. Ho SK, Coman RM, Bunger JC, Rose SL, O'Brien P, Munoz I, Dunn BM, Sleasman JW, Goodenow MM. 2008. Drug-associated changes in amino acid residues in Gag p2, p7NC, and p6Gag/p6Pol in human immunodeficiency virus type 1 (HIV-1) display a dominant effect on replicative fitness and drug response. Virology 378:272-281. https://doi.org/10 .1016/j.virol.2008.05.029.

27. Ibe S, Shibata N, Utsumi M, Kaneda T. 2003. Selection of human immunodeficiency virus type 1 variants with an insertion mutation in the p6gag and p6pol genes under highly active antiretroviral therapy. Microbiol Immunol 47:71-79. https://doi.org/10.1111/j.1348-0421.2003 .tb02788.x.
28. Whitehurst N, Chappey C, Petropoulos C, Parkin N, Gamarnik A. 2003. Polymorphisms in $\mathrm{p} 1-\mathrm{p} 6 / \mathrm{p}^{*}$ of HIV type 1 can delay protease autoprocessing and increase drug susceptibility. AIDS Res Hum Retroviruses 19:779-784. https://doi.org/10.1089/088922203769232575.

29. Barrie KA, Perez EE, Lamers SL, Farmerie WG, Dunn BM, Sleasman JW, Goodenow MM. 1996. Natural variation in HIV-1 protease, Gag p7 and p6, and protease cleavage sites within Gag/Pol polyproteins: amino acid substitutions in the absence of protease inhibitors in mothers and children infected by human immunodeficiency virus type 1. Virology 219:407-416. https://doi.org/10.1006/viro.1996.0266.

30. Song HY, Meng FZ, Xing H, Ruan HY, Li PX, Xin LR, Ma FP, Peng $H$, Shao Y. 2007. Analysis of HIV-1 CRF07_BC gag p6 sequences indicating novel deletions in the central region of p6. Arch Virol 152:1553-1558. https:// doi.org/10.1007/s00705-007-0973-6.

31. Chatterjee A, Mridula P, Mishra RK, Mittal R, Hosur RV. 2005. Folding regulates autoprocessing of HIV-1 protease precursor. J Biol Chem 280: 11369-11378. https://doi.org/10.1074/jbc.M412603200.

32. Ishima R, Torchia DA, Louis JM. 2007. Mutational and structural studies aimed at characterizing the monomer of HIV-1 protease and its precursor. J Biol Chem 282:17190-17199. https://doi.org/10.1074/jbc .M701304200.

33. Louis JM, Clore GM, Gronenborn AM. 1999. Autoprocessing of HIV-1 protease is tightly coupled to protein folding. Nat Struct Mol Biol 6:868-875. https://doi.org/10.1038/12327.

34. Sadiq SK, Noé F, De Fabritiis G. 2012. Kinetic characterization of the critical step in HIV-1 protease maturation. Proc Natl Acad Sci U S A 109:20449-20454. https://doi.org/10.1073/pnas.1210983109.

35. Pettit S, Clemente J, Jeung J, Dunn B, Kaplan A. 2005. Ordered processing of the human immunodeficiency virus type 1 GagPol precursor is influenced by the context of the embedded viral protease. J Virol 79:10601-10607. https://doi.org/10.1128/JVI.79.16.10601-10607.2005.

36. Liao W-H, Wang C-T. 2004. Characterization of human immunodeficiency virus type 1 Pr160gag-pol mutants with truncations downstream of the protease domain. Virology 329:180-188. https://doi.org/10.1016/j.virol .2004.08.010.

37. Zábranský A, Hunter E, Sakalian M. 2002. Identification of a minimal HIV-1 Gag domain sufficient for self-association. Virology 294:141-150. https://doi.org/10.1006/viro.2001.1315.

38. Burniston MT, Cimarelli A, Colgan J, Curtis SP, Luban J. 1999. Human immunodeficiency virus type 1 Gag polyprotein multimerization requires the nucleocapsid domain and RNA and is promoted by the capsid-dimer interface and the basic region of matrix protein. J Virol 73:8527-8540.

39. Rosé JR, Salto R, Craik CS. 1993. Regulation of autoproteolysis of the HIV-1 and HIV-2 proteases with engineered amino acid substitutions. J Biol Chem 268:11939-11945.

40. Mildner AM, Rothrock DJ, Leone JW, Bannow CA, Lull JM, Reardon IM, Sarcich JL, Howe WJ, Tomich C-SC. 1994. The HIV-1 protease as enzyme and substrate: mutagenesis of autolysis sites and generation of a stable mutant with retained kinetic properties. Biochemistry 33:9405-9413. https://doi.org/10.1021/bi00198a005.

41. Dautin N, Karimova G, Ladant D. 2003. Human immunodeficiency virus (HIV) type 1 transframe protein can restore activity to a dimerizationdeficient HIV protease variant. J Virol 77:8216-8226. https://doi.org/10 .1128/JVI.77.15.8216-8226.2003.

42. Louis JM, Nashed NT, Parris KD, Kimmel AR, Jerina DM. 1994. Kinetics and mechanism of autoprocessing of human immunodeficiency virus type 1 protease from an analog of the Gag-Pol polyprotein. Proc Natl Acad Sci U S A 91:7970-7974. https://doi.org/10.1073/pnas.91.17.7970.

43. Pettit S, Gulnik S, Everitt L, Kaplan A. 2003. The dimer interfaces of protease and extra-protease domains influence the activation of protease and the specificity of GagPol cleavage. J Virol 77:366-374. https:// doi.org/10.1128/JVI.77.1.366-374.2003.

44. Quillent C, Borman AM, Paulous S, Dauguet C, Clavel F. 1996. Extensive regions of pol are required for efficient human immunodeficiency virus polyprotein processing and particle maturation. Virology 219:29-36. https://doi.org/10.1006/viro.1996.0219.

45. Zybarth G, Carter C. 1995. Domains upstream of the protease (PR) in human immunodeficiency virus type $1 \mathrm{Gag}$-Pol influence PR autoprocessing. J Virol 69:3878-3884.

46. Leiherer A, Ludwig C, Wagner R. 2009. Uncoupling human immunodeficiency virus type $1 \mathrm{gag}$ and pol reading frames: role of the transframe protein $\mathrm{p}^{*}$ in viral replication. J Virol 83:7210-7220. https://doi.org/10 .1128/JVI.02603-08. 
47. Agniswamy J, Sayer JM, Weber IT, Louis JM. 2012. Terminal interface conformations modulate dimer stability prior to amino terminal autoprocessing of HIV-1 protease. Biochemistry 51:1041-1050. https://doi .org/10.1021/bi201809s.

48. Huang L, Li Y, Chen C. 2011. Flexible catalytic site conformations implicated in modulation of HIV-1 protease autoprocessing reactions. Retrovirology 8:79. https://doi.org/10.1186/1742-4690-8-79.

49. Tang C, Louis JM, Aniana A, Suh J-Y, Clore GM. 2008. Visualizing transient events in amino-terminal autoprocessing of HIV-1 protease. Nature 455: 693-696. https://doi.org/10.1038/nature07342.

50. Page KA, Landau NR, Littman DR. 1990. Construction and use of a human immunodeficiency virus vector for analysis of virus infectivity. J Virol 64:5270-5276.

51. Zhang Y, Qian H, Love Z, Barklis E. 1998. Analysis of the assembly function of the human immunodeficiency virus type 1 gag protein nucleocapsid domain. J Virol 72:1782-1789.

52. Loriaux MM, Rehfuss RP, Brennan RG, Goodman RH. 1993. Engineered leucine zippers show that hemiphosphorylated CREB complexes are transcriptionally active. Proc Natl Acad Sci U S A 90:9046-9050. https:// doi.org/10.1073/pnas.90.19.9046.

53. Burns JC, Friedmann T, Driever W, Burrascano M, Yee J. 1993. Vesicular stomatitis virus $\mathrm{G}$ glycoprotein pseudotyped retroviral vectors: concentration to very high titer and efficient gene transfer into mammalian and nonmammalian cells. Proc Natl Acad Sci U S A 90:8033-8037. https:// doi.org/10.1073/pnas.90.17.8033.

54. Chen YL, Ts'ai PW, Yang CC, Wang CT. 1997. Generation of infectious virus particles by transient co-expression of human immunodeficiency virus type 1 gag mutants. J Gen Virol 78:2497-2501. https://doi.org/10 .1099/0022-1317-78-10-2497.

55. Ferris AL, Hizi A, Showalter SD, Pichuantes S, Babe L, Craik CS, Hughes SH. 1990. Immunologic and proteolytic analysis of HIV-1 reverse transcriptase structure. Virology 175:456-464. https://doi.org/10.1016/0042 $-6822(90) 90430-Y$

56. Hizi A, McGill C, Hughes SH. 1988. Expression of soluble, enzymatically active, human immunodeficiency virus reverse transcriptase in Escherichia coli and analysis of mutants. Proc Natl Acad Sci U S A 85: 1218-1222. https://doi.org/10.1073/pnas.85.4.1218.

57. Chiang C-C, Wang S-M, Pan Y-Y, Huang K-J, Wang C-T. 2010. A single amino acid substitution in HIV-1 reverse transcriptase significantly reduces virion release. J Virol 84:976-982. https://doi.org/10.1128/JVI .01532-09. 